hep-th/9609159, IASSNS-HEP-96-97

\title{
PHYSICAL INTERPRETATION OF CERTAIN STRONG COUPLING SINGULARITIES
}

\author{
Edward Witten五 \\ School of Natural Sciences, Institute for Advanced Study \\ Olden Lane, Princeton, NJ 08540, USA
}

\begin{abstract}
We interpret certain strong coupling singularities of the $E_{8} \times E_{8}$ heterotic string on K3 in terms of exotic six-dimensional theories in which $E_{8}$ is a gauge symmetry. These theories are closely related to theories obtained at small instanton singularities, which have $E_{8}$ as a global symmetry.
\end{abstract}

September, 1996

1 Research supported in part by NSF Grant PHY-9513835. 


\section{Introduction}

By considering Type IIB superstring theory on K3, near an $A-D-E$ singularity, one obtains an exotic and previously unknown six-dimensional theory [1] from which gravity decouples. (The same theory can be constructed via parallel five-branes [2].) The limiting theory is a flat space, interacting, super-Poincaré-invariant, theory which may well obey the axioms of local field theory but whose existence is certainly not predicted by any standard field-theoretic construction. (In fact, known constructions above four dimensions appear to give only free field theories.) One indication of the interest of this particular exotic theory is that its existence has $S$-duality of four-dimensional $N=4$ super-Yang-Mills theory (for $A-D-E$ gauge groups) as a corollary [1].

Other similarly exotic theories may be constructed, for example, by taking suitable limits of heterotic string compactification on K3. By considering a small $E_{8}$ instanton, one gets a six-dimensional theory [3, 4] in which $E_{8}$ appears as a global symmetry group. This theory has extremely rich properties, as shown in [6]8]. By increasing the heterotic string coupling to a critical value [9] one obtains a somewhat analogous critical point, most effectively studied using $F$-theory [10,11]. These theories have strings, which can be constructed approximately as solitons [12], whose tension vanishes at the critical point.

In fact, the K3 constructions each give several theories. By considering $n$ nearly coincident small $E_{8}$ instantons, one gets a theory that we will call $V_{n}$. For reasons that we will review in section two, $V_{n}$ is a theory with $E_{8}$ as a global symmetry group. To obtain exotic theories with local gauge symmetry in six dimensions, we can turn to the strong coupling singularities. Consider the $E_{8} \times E_{8}$ heterotic string on K3, with instanton numbers $(24-n, n)$ in the two factors. If $n=12$ there is no strong coupling singularity [9]; for $n \neq 12$ there is (upon possibly exchanging the two factors) no loss of generality in assuming that $n<12$. The $(24-n, n)$ theory with $n<12$ has for its strong coupling singularity a theory that we will call $T_{n}$.

We will argue that the $T_{n}$ 's should be interpreted as theories with local $E_{8}$ gauge symmetry. Among these, we will interrpret $T_{0}$ as a sort of (almost) "pure" gauge theory with "no charged matter fields," Given such a theory, one can try to couple to "matter," that is to a theory with $E_{8}$ as a global symmetry. In six dimensions, the only theories we know with $E_{8}$ global symmetry are the $V_{n}$ 's; we will argue that at least heuristically, the theories $T_{n}$ for $1 \leq n \leq 11$ can be interpreted as a "gauging" of $V_{n}$ by coupling to the (almost) "pure gauge theory" $T_{0}$. 


\section{Review Of Small Instanton Theories}

First, we reconsider the theory that arises from a small $E_{8}$ instanton in heterotic string compactification on $\mathbf{R}^{6} \times \mathrm{K} 3$.

A small instanton near a point $p \in \mathrm{K} 3$ can be interpreted as a five-brane whose worldvolume fills out the six-manifold $Q=\mathbf{R}^{6} \times\{p\}$. Certain modes propagate on $Q$. To claim that the theory on $Q$ is a flat space theory with $E_{8}$ as a global symmetry, the key point is to show that both the gravitons and the $E_{8}$ gauge bosons can be made to decouple from the theory on $Q$, to any desired precision. This may be done simply by increasing the volume of the K3. The graviton and gauge boson wave functions then spread all over K3, and as the $\mathrm{K} 3$ grows, they decouple from the theory on $Q$. The fact that the $E_{8}$ gauge bosons decouple from the theory on $Q$ means that in studying this theory, $E_{8}$ can be treated as a global symmetry. The fact that the graviton decouples means that this is likewise a theory in which the Poincaré group is a global symmetry and there is no dynamical gravity, something which we have loosely described by calling the theory a flat space theory.

The small instanton theory, which we will call $V_{1}$, has a Higgs branch with 29 massless hypermultiplets (representing the size and $E_{8}$ orientation of the instanton), and a Coulomb branch with one massless tensor multiplet (corresponding to a situation in which the instanton has been converted to an $M$-theory five-brane). One could add a thirtieth massless hypermultiplet, representing the instanton center of mass, but this would be a decoupled free field and we choose not to include it.

By considering $n$ nearly coincident small $E_{8}$ instantons, one can construct further theories that we will call $V_{n}$. $V_{n}$ has a Higgs branch with $30 n-1$ massless hypermultiplets (which parametrize the sizes, $E_{8}$ orientations, and relative positions of the instantons), a Coulomb branch with $n$ massless tensor multiplets (the branch with $n M$-theory fivebranes), and various mixed branches with some of each. Notice that on the Higgs branch, if we consider a situation in which the instantons are far apart compared to their sizes, then the theory $V_{n}$ reduces to $V_{1}^{n}$ ( $n$ copies of $V_{1}$ ) plus $n-1$ free massless hypermultiplets (the relative positions of the instantons). Other analogous reductions arise in limits in which some but not all of the separations between instantons are large compared to the instanton sizes.

\section{Search For Gauge Symmetry}

Now if we want to find an analogous theory with $E_{8}$ as a gauge symmetry, we must find a singularity at which the $E_{8}$ gauge bosons do not decouple. One option is the strong 
coupling singularity of the $E_{8} \times E_{8}$ heterotic string with instanton numbers $(24-n, n)$, $n \leq 12$. Since it is hard to understand this singularity from the heterotic string point of view, it is not obvious from this point of view whether or not the gauge bosons are decoupled from whatever theory may emerge at the singularity. It is likewise not clear whether gravity is so decoupled.

What settles this question is the approach via $F$-theory [10]. The $(24-n, n)$ heterotic string is equivalent to $F$-theory on the Hirzebruch surface $\mathbf{F}_{12-n}$. In this description, the strong coupling singularity is localized on a curve $C \subset \mathbf{F}_{12-n}$. ( $C$ is the exceptional section of the Hirzebruch surface, with $C \cdot C=-12+n$.) Since the graviton wave functions are meanwhile spread over the whole Hirzebruch surface, it follows that gravity is decoupled from the theory that emerges at the singularity, so that this theory (if it is a field theory at all) is a flat space quantum field theory. On the other hand, according to [10, the gauge bosons of one of the two $E_{8}$ 's (namely the one in which the instanton number is $n$ ) are localized on the same curve $C$, leading us to expect that they are not decoupled from whatever physics is supported on $C$. Therefore, the theory - which we will call $T_{n}$ - associated with the strong coupling singularity of the $(24-n, n)$ heterotic string is potentially an $E_{8}$ gauge theory.

One may wonder whether in some situation gravity would fail to decouple from the modes that become strongly coupled at such a singularity, giving some sort of exotic phase of quantum general relativity with novel long distance behavior. Whether this is possible is not clear.

\section{Identifying The "Matter Content"}

Going back to the $T_{n}$ 's, we would like to say more about their "matter content." What is the unbroken gauge group in the theory $T_{n}$ ? This depends on where we are in moduli space. For any given value of $n$, in the $(24-n, n)$ heterotic string compactification on K3, there is a generic unbroken subgroup of $E_{8}$ (that is, of the "second" $E_{8}$ with instanton number $n$ ). We will call this generic unbroken group $G_{n}$. For instance (on the known branches of the moduli spaces), $G_{0}=G_{1}=G_{2}=G_{3}=E_{8}, G_{4}=E_{7}, G_{8}=S O(8)$, $G_{9}=S U(3)$, and $G_{10}$ and $G_{11}$ are trivial. (We stop the list at $n=11$, of course, since that is the last example for the strong coupling singularity that we are studying here.) On the other hand, at special loci in moduli space, it is possible to restore part or all of the $E_{8}$. 
We interpret this to mean that in some sense the $T_{n}$ theories are $E_{8}$ gauge theories coupled to matter. We propose that the $T_{0}$ theory, in which the $E_{8}$ is always unbroken, is roughly comparable to a "pure gauge theory." 2 This statement requires some explanation. In addition to the $E_{8}$ vector multiplets, the $T_{0}$ theory has a massless tensor multiplet (whose scalar parametrizes the heterotic string coupling constant) with a moduli space $\mathbf{R}^{+}$. The critical point is the endpoint of the $\mathbf{R}^{+}$, and at that point, since the physics is not free in the infrared, the particle spectrum and gauge group are likely to be ill-defined. However, at a generic point on $\mathbf{R}^{+}$, away from the endpoint, the physics is infrared-free, the spectrum makes sense, and the gauge group is well-defined and equal to $E_{8}$. In calling the theory an (almost) "pure gauge theory," we mean simply that away from the critical point, there is no charged matter; the generic massless spectrum consists of the $E_{8}$ vector multiplet plus one tensor multiplet.

Now, if the $T_{0}$ theory is thought of as an $E_{8}$ gauge theory, one might expect to be able to couple it to a "matter system," that is a field theory with $E_{8}$ global symmetry, which one might try to gauge. One set of theories with global $E_{8}$ symmetry are the small instanton theories $V_{n}, n \geq 1$. We propose that the $T_{n}$ theories for $n=1, \ldots, 11$ are obtained by "gauging" the $V_{n}$, that is by coupling them to the "pure gauge theory" $T_{0}$. (The fact that the $T_{n}$ series apparently ends at $n=11$ would from this point of view mean that for $n>11$ one has "too much matter" to obtain a well-defined theory; the bound on the amount of matter would be analogous to the familiar asymptotic freedom bound in four dimensions, or the less familiar bound found in five dimensions in [5].)

As a check on this, we may try to show that the theory obtained by "gauging" $V_{n}$ would have the same generic unbroken gauge group on the Higgs branch as the $(24-n, n)$ heterotic string. In fact, this is almost a tautology. If we gauge $V_{n}$, the moduli space of the Higgs branch is obtained by starting with the moduli space $\mathcal{M}_{n}$ of $n E_{8}$ instantons on $\mathbf{R}^{4}$, and then taking the "hyper-Kahler quotient" of $\mathcal{M}_{n}$ by $E_{8}$. (The hyper-Kahler quotient is obtained by setting the $D$-fields to zero and then dividing by $E_{8}$. $D$-fields, sometimes called the components of the hyper-Kahler moment map, are defined for any hyper-Kahler manifold - in this case the Higgs branch of $V_{n}$ - with a simple group action - in this case $E_{8}$. One must set the $D$-fields to zero to find a supersymmetric vacuum because the potential energy of the supersymmetric gauge theory is a multiple of $\vec{D}^{2}$.) On

2 The $T_{1}, T_{2}$, and $T_{3}$ theories also have always unbroken $E_{8}$, but will be given a slightly different interpretation momentarily. 
the other hand, if we consider $n$ almost coincident small $E_{8}$ instantons on K3, the moduli space of such objects is precisely the same as the hyper-Kahler quotient of $\mathcal{M}_{n}$ by $E_{8}$ 目 (assuming that one suppresses the overall center of mass position of the instantons, which is parametrized by $\mathbf{R}^{4}$ in one case and $\mathrm{K} 3$ in the other). Therefore, not only does the theory obtained heuristically by "gauging" $V_{n}$ have for its generic unbroken gauge group on the Higgs branch the group $G_{n}$ that appears in the $(24-n, n)$ heterotic string; the Higgs branches actually coincide.

The fact that the theories $T_{i}$ for $i=1,2,3$ have the same unbroken gauge group as $T_{0}$ is now clear intuitively: they have massless charged matter fields but not enough of them to generate a Higgs branch.

Now let us ask what is the smallest value of $n$ for which complete Higgsing of $E_{8}$ is possible in the $T_{n}$ theory. From what has been said, this is the same as the smallest value of $n$ for which complete Higgsing is possible in the $(24-n, n)$ heterotic string, namely $n=10$.

Now consider the theory $T_{11}$. We interpret this as the "gauging" of the theory $V_{11}$ of 11 small instantons. In a suitable limit (with one small instanton far from the other ten) $V_{11}$ reduces to $V_{1} \times V_{10}$ (with an additional and irrelevant massless free hypermultiplet that parametrizes the relative separation of the ten instantons from the eleventh one). Therefore, in this limit, $T_{11}$ reduces to the "gauging" of $V_{1} \times V_{10}$. The "gauging" of $V_{10}$ gives a theory in which complete Higgsing is possible. Upon going to a region of $V_{10}$ moduli space in which this complete Higgsing occurs, the $E_{8}$ gauge bosons all get mass. The low energy theory in this region consists of some infrared-free massless hypermultiplets plus a copy of the theory $V_{1}$, which near its critical point is an interacting theory.

We have deduced, therefore, that the theory $T_{11}$, on a suitable part of its Higgs branch, reduces to the theory $V_{1}$. This corresponds to a familiar fact [1]: the strong coupling singularity of the $(13,11)$ heterotic string is actually governed by the small instanton theory $V_{1}$.

This then is perhaps the one fact that we have explained in this paper: the value of $n$ at which the $(24-n, n)$ heterotic string has $V_{1}$ for its strong coupling singularity (namely

3 Since $\mathrm{K} 3$ is compact, one automatically divides by $E_{8}$ in constructing instanton moduli space on K3. Supersymmetry relates the operation of dividing by $E_{8}$ to that of setting to zero the $D$ fields, which therefore necessarily is implicit in the moduli problem of instantons on K3. It is possible by standards arguments to show explicitly how vanishing of the $D$ fields comes in in the small instanton limit, but this will not be done here. 
$n=11$ ) is one more than the smallest value (namely $n=10$ ) at which complete Higgsing is possible. 


\section{References}

[1] E. Witten, "Some Comments On String Dynamics," hepth/9507121.

[2] A. Strominger, "Open p-Branes," hepth/9512059.

[3] O. Ganor and A. Hanany, "Small $E_{8}$ Instantons And Tensionless Noncritical Strings," hepth/9602120.

[4] N. Seiberg and E. Witten, "Comments On String Dynamics In Six Dimensions," hepth/9603003.

[5] N. Seiberg, "Five-Dimensional SUSY Field Theories, Non-trivial Fixed Points, and String Dynamics," hepth/9608111.

[6] D. Morrison and N. Seiberg, "Extremal Transitions And Five-Dimensional Supersymmetric Field Theories," hepth/9609070.

[7] M. Douglas, S. Katz, and C. Vafa, "Small Instantons, Del Pezzo Surfaces, And Type $I^{\prime}$ Theory," hepth/9609071.

[8] O. Ganor, "Toroidal Compactification Of Heterotic 6D Non-Critical Strings Down To Four Dimensions," hepth/9608109.

[9] M. J. Duff, R. Minasian, and E. Witten, "Evidence For Heterotic/Heterotic Duality," hepth/9601036.

[10] D. Morrison and C. Vafa, "Compactification of F-Theory On Calabi-Yau Threefolds, I, II," hepth/9602114,9603161.

[11] E. WItten, "Phase Transitions In $M$-Theory and F-Theory," hepth/9603150.

[12] M. Duff, H. Lu, and C. N. Pope, "Heterotic Phase Transitions And Singularities Of The Gauge Dyonic String," hepth/9603037, 